\title{
Noninvasive electron microscopy with interaction-free quantum measurements
}

\author{
William P. Putnam and Mehmet Fatih Yanik* \\ Department of Electrical Engineering and Computer Science and Research Laboratory of Electronics, \\ Massachusetts Institute of Technology, Cambridge, Massachusetts 02139, USA
}

(Received 20 January 2009; published 23 October 2009)

\begin{abstract}
We propose the use of interaction-free quantum measurements with electrons to eliminate sample damage in electron microscopy. This might allow noninvasive molecular-resolution imaging. We show the possibility of such measurements in the presence of experimentally measured quantum decoherence rates and using a scheme based on existing charged particle trapping techniques.
\end{abstract}

DOI: 10.1103/PhysRevA.80.040902

Electron microscopy has significantly impacted many areas of science and engineering due to its unprecedented atomic and molecular resolution. Yet, the imaging of biological and other sensitive specimens has been limited because of sample damage induced by the energetic electrons necessary for imaging. The radiation dose received by a specimen during imaging with an electron microscope operating under typical conditions is comparable to the irradiation from a 10 megaton hydrogen bomb exploded about thirty meters away [1]. Despite progress in hydrated environmental chambers for viewing biological specimens in their native state $[2,3]$, when exposed to such energetic electrons, sensitive specimens experience substantial mass loss, modification of chemical bonding, or other structural damage $[2,4]$.

At first sight, one might conclude that any measurement requires physical interaction with the measured object. Yet, it has been shown that a non-transmitting object in the arm of an optical interferometer can modify the interference of a single photon in such a way that the presence of the object can be detected without interaction (i.e., energy exchange) between the photon and the object [5-9]. Such interaction-free measurements have been employed in optical microscopy with photons [10] but never previously considered with electrons. Gabor, who investigated several electron microscopy techniques, concluded in his famous review [11]: "The fundamental limitation of electron interferometers... is the destruction of the object by the exploring agent, and in this respect electron interferometers appear to be neither better nor worse than other instruments." Here, we propose and show the possibility of interaction-free measurements with electrons in the presence of previously measured quantum decoherence rates and using a scheme based on charged particle trapping techniques. Use of this quantum electron microscopy (QEM) technique might dramatically reduce sample damage in electron microscopy while achieving molecular-level resolution.

We consider two ring-shaped electron guides each with radius $R$ and vertically stacked with a separation of $\Delta z$ as shown in Fig. 1(a). A potential $U_{\text {eff }}(r, z)$ provides confinement in the $\vec{r}$ and $\vec{z}$ directions and guides electrons in circular paths along the rings. The guiding potential $U_{\text {eff }}(r, z)$ also couples the two ring shaped guides with a double-well potential in the $\vec{z}$ direction.

\footnotetext{
*yanik@mit.edu
}

PACS number(s): 07.78.+s, 42.50.Dv

Imagine an electron propagating around the rings. The electron wave function can be separated into an angular $\theta$-dependent portion and a planar $(r, z)$-dependent part. Due to the double-well potential, the two lowest-energy states of

a.

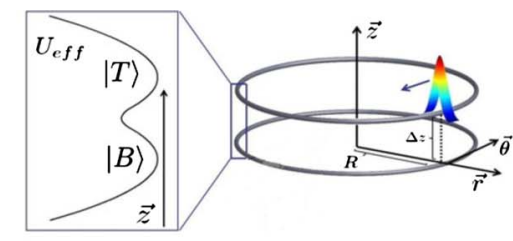

b.

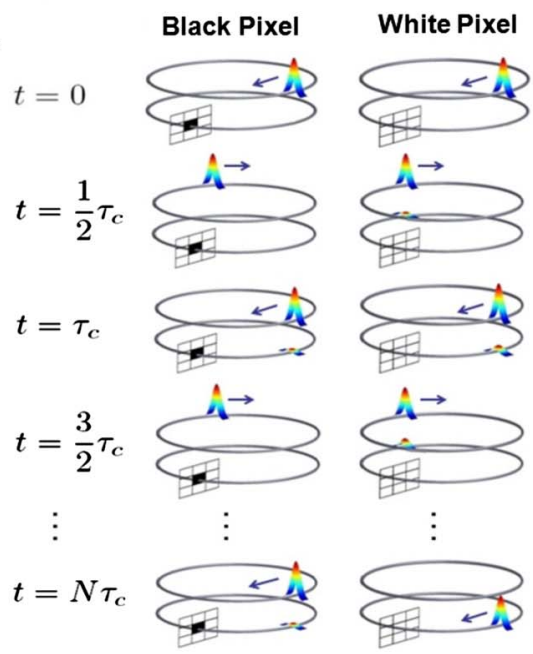

c.

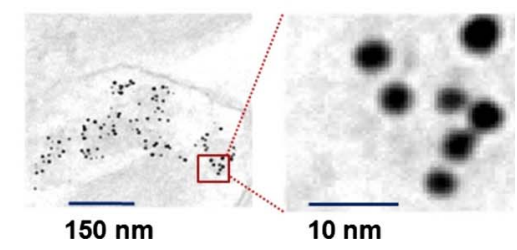

FIG. 1. (Color online) (a) Coupled electron ring guides. The wave packet illustrates the amplitude of the circulating electron. The guide potential $U_{\text {eff }}(r, z)$ couples the localized electron states $|T\rangle$ and $|B\rangle$ in a double-well potential. (b) Interaction-free imaging with electrons. The grid in the lower ring is the object being imaged, which is composed of opaque and transparent regions (i.e., black and white pixels). (c) Example of high-contrast TEM imaging at $100 \mathrm{KeV}$. Gold nanoparticles labeled with antibody against vesicular monoamine transporter appear as black dots while the rest of the tissue in the background is significantly transparent to the incident electrons. The image contrast is reduced to make the background visible. Image courtesy of Kathryn Commons. 
the electron in the $r-z$ plane (i.e., the transverse ground and first excited states) correspond to a symmetric state $\left|\Psi_{s}\right\rangle$ with energy $E_{s}$ and an antisymmetric state $\left|\Psi_{a}\right\rangle$ with energy $E_{a}$. States which correspond to spatial localization of the electron in the top ring and the bottom ring can be expressed as $|T\rangle=\left(\left|\Psi_{s}\right\rangle+\left|\Psi_{a}\right\rangle\right) / \sqrt{2}$ and $|B\rangle=\left(\left|\Psi_{s}\right\rangle-\left|\Psi_{a}\right\rangle\right) / \sqrt{2}$, respectively.

When the energy splitting $2 \hbar \Delta \equiv E_{a}-E_{s}$ is sufficiently small $\left(\ll 2 \hbar \omega_{0}=E_{a}+E_{s}\right)$, the double well can be approximated as a two-state system. The Hamiltonian can then be written in the $|T\rangle,|B\rangle$ basis,

$$
H=\left(E_{\theta}+\hbar \omega_{0}\right) I-\hbar \Delta \sigma_{x},
$$

where $\hbar \omega_{0}$ is the ground state energy of each well separately and $E_{\theta}$ is the kinetic energy due to the electron motion in the $\vec{\theta}$ direction (the energy spread, $\delta E_{\theta}$, of the electron wave packet can be neglected for the TEM parameters of interest $\left.E_{\theta}=100 \mathrm{KeV}, \delta E_{\theta} \sim 0.1 \mathrm{eV}\right)$.

For simplicity, we will initially treat this system as isolated from its environment. Solving for the density-matrix time evolution, we recover the well-known result for twostate systems: the circularly propagating electron in Fig. 1(a), initially prepared in a localized state in the top ring, undergoes undamped oscillations between the states $|T\rangle$ and $|B\rangle$. The time-dependent probabilities of the electron occupying the top versus the bottom rings are then given by $P_{T}(t)$ $=\cos ^{2}(\Delta t)$ and $P_{B}(t)=\sin ^{2}(\Delta t)$, respectively. Defining $\tau_{C}$ as the time required for the electron to complete one circulation about the rings, it takes the electron $N=\pi /\left(2 \Delta \tau_{C}\right)$ circulations to transfer from one ring to the other.

Consider the setup in Fig. 1(b): an electron is injected into the top ring (initially prepared in $|T\rangle$ ), and an object composed of opaque and transparent regions (i.e., pseudoblack and white pixels) crosses the electron's path in the bottom ring. Opaque (transparent) regions have a probability of electron transmission near zero (one). In high-energy TEM, commonly used staining or immunolabeling with heavy metal solutions or metal nanoparticles allows one to achieve significantly high-contrast transmission [Fig. 1(c)], where metals almost completely block electron transmission while the rest of the thin specimen becomes highly transparent to electrons at high kinetic energies [12]. Thus, the opaque and transparent idealization can be well approximated in transmission electron microscopy in contrast to standard light microscopy. With a transparent region of the object in the bottom ring [i.e., a white pixel in Fig. 1(b)], the evolution of the circulating electron wave packet is unaffected. After $N$ circulations the electron transfers entirely from state $|T\rangle$ to state $|B\rangle$, i.e., the probability of measuring the electron in $|B\rangle$ after $N$ circulations given the presence of a transparent region is $P(B \mid$ transparent $)=P_{B}\left(N \tau_{C}\right)=1$.

If an opaque region of the object [i.e., a black pixel in Fig. 1(b)] blocks the electron's pathway, however, the coherent transfer of the electron between the rings is prevented. After being injected into the top ring the electron begins to evolve from $|T\rangle$ to $|B\rangle$, but after a time $\tau_{C}$ the presence of the opaque region forces a measurement on the spatial state of the electron. If $\Delta \tau_{C}$ is small (i.e., $N$ is large), the electron's wave function is projected back to the top ring with a high probability of $P_{T}\left(\tau_{C}\right)=\cos ^{2} \Delta \tau_{C} \approx 1-\pi^{2} / 4 N^{2}$. With each circulation around the ring, this measurement process is repeated, and after $N$ circulations the electron remains in $|T\rangle$ with a probability of $P_{T}\left(\tau_{C}\right)^{N}=\cos ^{2 N} \Delta \tau_{C} \approx 1-\pi^{2} / 4 N$. This is just a discrete quantum Zeno effect [8]. Thus, after $N$ circulations and given the presence of an opaque region, the probability of measuring the electron in $|T\rangle$ is $P(T \mid$ opaque $)=P_{T}\left(\tau_{C}\right)^{N} \approx 1-\pi^{2} / 4 N$, and the probability of the electron being scattered by the object is $P($ scat $)=1$ $-P(T \mid$ opaque $) \approx \pi^{2} / 4 N$.

By measuring which ring the electron is in after $N$ circulations, the presence of an opaque or transparent region of an object in the bottom ring can then be determined with vanishing probability of scattering from the object. An image of an object composed of opaque and transparent regions can then be generated by rastering the object across the electron's path in the bottom ring where the electron beam width in the $r-z$ plane dictates the pixel resolution.

The accuracy $\eta$ of this interaction-free imaging can be defined as the probability of correctly determining the presence of an opaque or transparent region after $N$ circulations without scattering [7]. Assuming the a priori probabilities of a region being opaque or transparent are equal, i.e., $P($ opaque $)=P($ transparent $)=1 / 2$, the accuracy becomes $\eta$ $=\frac{1}{2}[P(T \mid$ opaque $)+P(B \mid$ transparent $)]$. For the system in our preceding discussion, which was isolated from its environment, the accuracy is then $\eta \approx 1-\pi^{2} / 8 N$. By making $N$ large, this accuracy can be made arbitrarily close to one: opaque and transparent regions can be distinguished with arbitrarily high probability without scattering. However, in practice the system is not completely isolated from its environment, and as we discuss next, the maximum accuracy can be close to but is always less than one.

Interactions between the trapped electron and the surrounding environment decohere the electron's spatial state. When the coupling to the environment is sufficiently weak and the correlation time of the interactions is small, a set of Bloch-type equations can be written for the time evolution of the system's reduced density matrix [13,14]. Solving these equations for an electron initially prepared in the top ring, the probabilities of an electron to be in the top versus the bottom ring can be found to be $P_{T}(t)=\frac{1}{2}(1$ $\left.+e^{-t / 2 \tau_{D}} \cos 2 \Delta^{\prime} t\right)$ and $P_{B}(t)=\frac{1}{2}\left(1-e^{-t / 2 \tau_{D}} \cos 2 \Delta^{\prime} t\right)$, respectively, where $\tau_{D}$ is the decoherence time (i.e., the time scale over which the electron loses its spatial coherence) and $\Delta^{\prime}$ is the modified tunneling rate, $\Delta^{\prime}=\sqrt{\Delta^{2}-1 / 16 \tau_{D}^{2}}$.

Using these expressions for $P_{B}(t)$ and $P_{T}(t)$, we find the accuracy in the presence of a decohering environment to be $\eta(N, \alpha) \approx 1 / 2^{N+1}\left[1+e^{-\alpha / 2} \cos (\pi / N)\right]^{N}+1 / 4\left(1+e^{-N \alpha / 2}\right)$. The dimensionless parameter $\alpha^{-1} \equiv \tau_{D} / \tau_{C}$ describes the decoherence strength. The probability of electron scattering by an opaque region (or the electron exposure reduction since exposure is proportional to scattering) after $N$ circulations (given the presence of an opaque region) can likewise be found to be

$$
P(\text { scat }) \approx 1-\frac{1}{2^{N}}\left[1+e^{-\alpha / 2} \cos (\pi / N)\right]^{N} .
$$




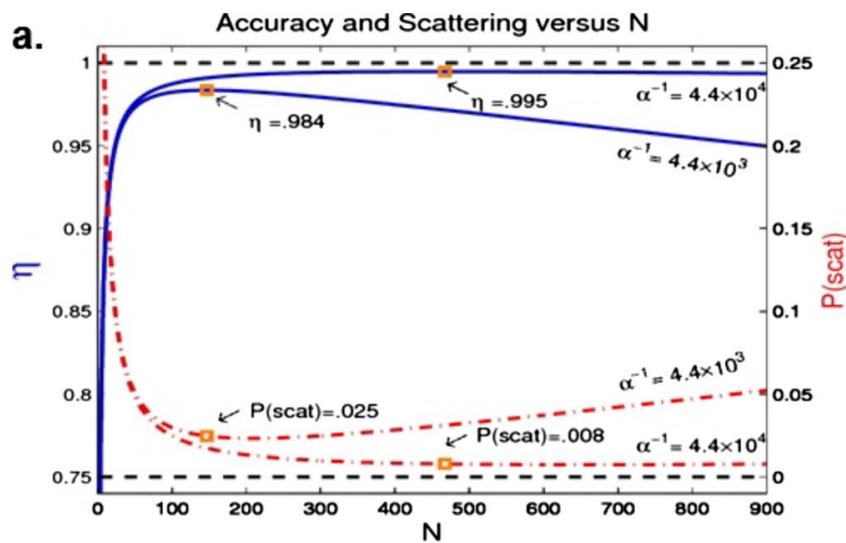

b.

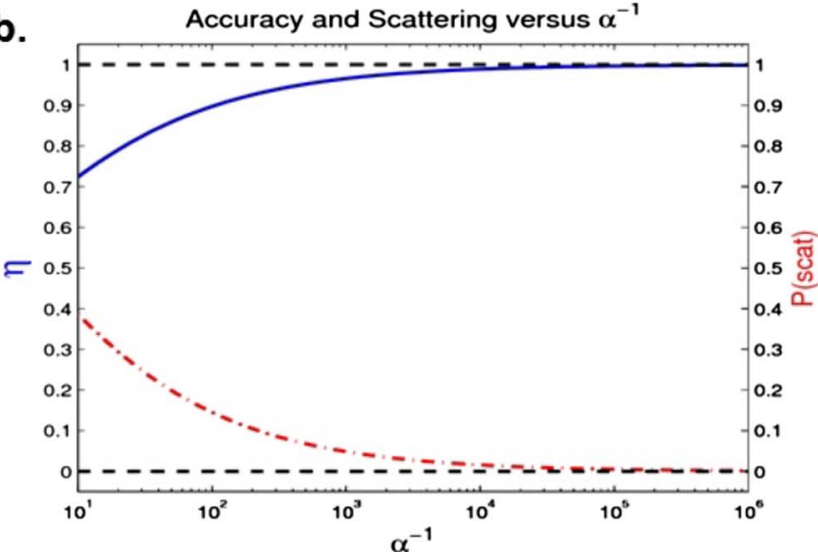

FIG. 2. (Color online) Accuracy of interaction-free imaging and probability of electron scattering from the imaged object in the presence of decoherence. (a) The solid (blue) and dash-dot (red) curves are plots of the imaging accuracy $\eta$ and the probability of scattering $P$ (scat) versus $N$, respectively. The squares show points of maximum accuracy and corresponding values of scattering probability. The $\alpha^{-1}$ values $4.4 \times 10^{3}$ and $4.4 \times 10^{4}$ correspond to 100 $\mathrm{KeV}$ electrons circulating a ring of radius $R=1 \mathrm{~cm}$ and $1 \mathrm{~mm}$, respectively, with a decoherence time of $\tau_{D}=1.7 \mu \mathrm{s}$. (b) The solid (blue) and dash-dot (red) curves are, respectively, the maximum values of $\eta$ and the minimum values of $P$ (scat) as functions of $\alpha^{-1}$.

As illustrated in Fig. 2(a), in the presence of decoherence, the imaging accuracy has a maximum value less than one, and the probability of scattering has a minimum value greater than zero. For large $\alpha^{-1}$, environmental interactions become less influential and the system behaves more like the ideal one we discussed previously, i.e., the maximum $\eta$ approaches one and the minimum $P$ (scat) approaches zero as in Fig. 2(b). We next estimate $\eta$ and $P$ (scat) in the presence of previously reported electron decoherence rates and using an implementation of the ring shaped electron guides based on conventional charged-particle trapping techniques.

A commonly used charged particle trap is the linear Paul trap which applies radio-frequency $(\mathrm{RF})$ voltages to produce an oscillating quadrupole field and confine ion motion to an axis. Bending a linear Paul trap into a circle, a ring shaped trap can be made [15].

The Paul trap's quadrupole field can be created by a variety of electrode geometries, and recently planar linear Paul traps with all electrodes on a single surface were demonstrated [16]. If two such linear surface-electrode traps are

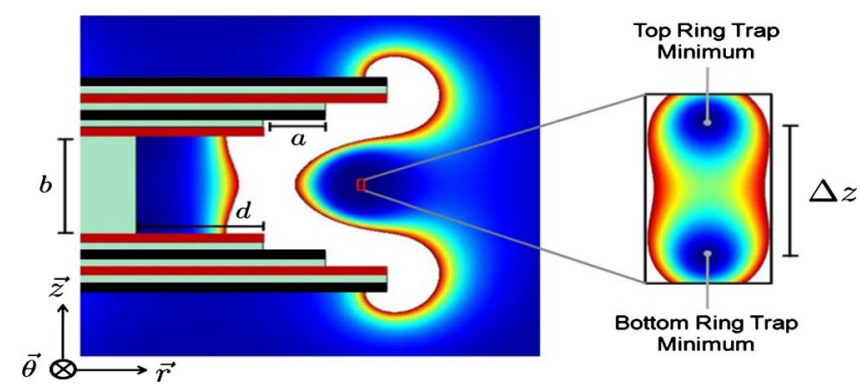

FIG. 3. (Color online) Multilayered structure equivalent to the $\mathrm{v}$-shaped trap arrangement but more suitable for microfabrication. Black and dark gray (red) rectangles are grounded and RF electrodes $(\mathrm{Au})$, respectively, and light gray (green) is insulating $\mathrm{SiO}_{2}$. The effective potential $U_{\text {eff }}(r, z)$ is superimposed on the structure; dark (blue) and light (red) shades are low and high potentials, respectively, and white regions have potentials $>20 \mathrm{meV}$. RF voltage in this example is driven at $10 \mathrm{GHz}$ with a magnitude of $2 \mathrm{~V}$. The dimensions are $b=48.5 \mu \mathrm{m}, a=24 \mu \mathrm{m}$, and $d=50 \mu \mathrm{m}$. The electrode width and spacing are $4 \mu \mathrm{m}$. The inset shows an expanded view of the double well. Near the trap minima the potential is harmonic with characteristic frequencies of $f_{r}, f_{z}=33 \mathrm{MHz}$, yielding a tunneling rate of $\Delta^{\prime}=2 \pi \times 14 \mathrm{MHz}$ and an electron spot size (i.e., resolution) of $19 \mathrm{~nm}$ and $1.4 \mu \mathrm{m}$ in the $\vec{r}$ and $\vec{z}$ directions, respectively (accounting for $B_{0}=1 \mathrm{~T}$ for $R=1 \mathrm{~mm}$ ).

arranged in a v-shaped configuration, a double-well potential can be formed between the two trapping minima. Bending the $\mathrm{v}$-shaped configuration into a circle, two ring traps coupled in a double well can be made. For a ring radius of $\approx 1 \mathrm{~mm}$ and typical TEM electron energies of $\approx 100 \mathrm{KeV}$, the necessary centripetal force for circulation is considerable. However, a magnetic field $B_{0}$ applied in the $\vec{z}$ direction (in this case $B_{0} \approx 1 \mathrm{~T}$ ) can supply the required force and converts our Paul trap arrangement to a combined trap, i.e., a hybrid Paul-Penning trap [17]. A cross section of the effective potential of the $\mathrm{v}$-shaped arrangement of linear surfaceelectrode traps is shown in Fig. 3.

The dimensions of the trap, in particular the height $b$ and the electrode width $a$ (refer to Fig. 3), can be varied to adjust the positions of the trap minima. The magnitude of the applied oscillating voltage can be used to tune the tightness of the trap, i.e., the trap's characteristic frequencies $f_{r}$ and $f_{z}$. Also since the tunneling depends only on the proximity of the two traps and their strengths, the influence of the electrode voltage noise on the tunneling time is small. For the example trap, a fluctuation of $100 \mu \mathrm{V}$ on one electrode results in only about $1 \%$ change in the tunneling time.

As electrons move in the traps, they induce image charges on the trap electrodes. The image charge distributions constitute which path information and result in the decoherence of the electron's spatial state to an incoherent mixture. Additionally, noise fields due to the surface charge fluctuations on the trap electrodes heat the trapped electron.

The time scale of image charge induced decoherence, $\tau_{d, \text { imag }}$, can be estimated using a model of two electron wave packets moving above an infinite conducting plate (i.e., one of the electrode surfaces) [18]. Image charge related decoherence time scales in such geometries have been experimen- 
tally measured for semiconductors [19]. A conservative scaling of these experimental results using the theoretically predicted form of electron decoherence [18] predicts $\tau_{\text {d,imag }}$ $\approx 11 \mu$ s for our system with a cryogenic electrode temperature of $T \approx 6 \mathrm{~K}$ (cryogenic surface electrode traps operating at $6 \mathrm{~K}$ have recently been demonstrated [20]).

A rough estimate for the heating (i.e., fluctuating field) induced decoherence time, $\tau_{d \text {,heat }}$, can be obtained by scaling heating rates measured in similar low-temperature surfaceelectrode traps [20]. Scaling to our trap parameters and using the relationship between decoherence and heating rate [21] yields a decoherence time of $\tau_{d \text {,heat }} \approx 2 \mu \mathrm{s}$. This conservative estimate is dependent on fabrication process [20] so future improvement may be possible.

The image charge and heating induced decoherence rates can be combined in a single rate, $\tau_{D}^{-1}=\tau_{d \text {,imag }}^{-1}+\tau_{d \text {,heat }}^{-1}$, describing the decoherence due to environmental interactions. Using the worst-case decoherence estimates above, a conservative estimate for the decoherence time is $\tau_{D} \approx 1.7 \mu \mathrm{s}$. Then $100 \mathrm{keV}$ electrons and a ring radius of either $1 \mathrm{~cm}$ or 1 mm gives $\alpha^{-1}$ values of $4.4 \times 10^{3}$ and $4.4 \times 10^{4}$, respectively. Referring to Fig. 2(a), we find corresponding scattering probabilities $P($ scat) (and accuracies $\eta$ ) of 0.03 (0.98) for $R$ $=1 \mathrm{~cm}$ and $0.01(0.99)$ for $R=1 \mathrm{~mm}$. Since sample exposure is proportional to electron scattering probability, this corresponds to two orders of magnitude reduction in sample exposure. Such a dramatic reduction in electron exposure could allow noninvasive imaging of molecular processes such as protein activity [2].
There is an intrinsic limitation to interaction-free imaging itself-semitransparencies (i.e., regions of an object that are neither opaque nor transparent). Semitransparent regions have a reduced interaction-free imaging accuracy and an increased probability of scattering [22]. However, as discussed above [Fig. 1(c)], transmission electron microscopy is often done in a high-contrast regime by adjusting incident electron energies and using labeling stains such as heavy metals and metal nanoparticles that strongly scatter electrons [12]. Thus, interaction-free electron measurements avoid the limitations of semitransparencies. Furthermore, a significant portion of tissue damage in TEM occurs due to electron scattering from the heavily labeled opaque regions, which would be avoided in our scheme due to the high efficiency of interaction-free measurements on such completely opaque regions.

Here, we have proposed and demonstrated the possibility of noninvasive measurements with electrons even in the presence of worst-case electron decoherence rate estimates using an interaction-free measurement scheme based on charged particle trapping techniques. Interaction-free quantum electron microscopy (QEM) can prevent sample exposure to highly energetic and destructive electrons in electron microscopy, which might allow noninvasive imaging of dynamic processes at molecular resolution and open frontiers in imaging.

We thank Nuh Gedik, Steve Quake, Mark Kasevich, and Peter Hommelhoff for feedback. This work was supported by David and Lucille Packard Foundation.
[1] D. T. Grubb and A. Keller, Proceedings of the Fifth European Regional Conferences on Electron Microscopy, Manchester, 1972 (unpublished), pp. 554-560.

[2] A. M. Glauert, J. Cell Biol. 63, 717 (1974).

[3] S. Thiberge et al., Proc. Natl. Acad. Sci. U.S.A. 101, 3346 (2004).

[4] R. Glaeser and K. Taylor, J. Microsc. 112, 127 (1978).

[5] A. Elitzur and L. Vaidman, Found. Phys. 23, 987 (1993).

[6] P. Kwiat, H. Weinfurter, T. Herzog, A. Zeilinger, and M. A. Kasevich, Phys. Rev. Lett. 74, 4763 (1995).

[7] T. Tsegaye et al., Phys. Rev. A 57, 3987 (1998).

[8] P. G. Kwiat et al., Phys. Rev. Lett. 83, 4725 (1999).

[9] J. Jang, Phys. Rev. A 59, 2322 (1999).

[10] A. G. White, J. R. Mitchell, O. Nairz, and P. G. Kwiat, Phys. Rev. A 58, 605 (1998).

[11] D. Gabor, Rev. Mod. Phys. 28, 260 (1956).
[12] M. A. Hayat, Principles and Techniques of Electron Microscopy (Cambridge University Press, Cambridge, England, 2000).

[13] A. J. Leggett et al., Rev. Mod. Phys. 59, 1 (1987).

[14] R. Harris and R. Silbey, J. Chem. Phys. 78, 7330 (1983).

[15] G. Birkl et al., Nature (London) 357, 310 (1992).

[16] S. Seidelin et al., Phys. Rev. Lett. 96, 253003 (2006).

[17] F. G. Major, Charged Particle Traps (Springer, New York, 2005).

[18] P. Machnikowski, Phys. Rev. B 73, 155109 (2006).

[19] P. Sonnentag and F. Hasselbach, Phys. Rev. Lett. 98, 200402 (2007).

[20] J. Labaziewicz et al., Phys. Rev. Lett. 100, 013001 (2008).

[21] Q. A. Turchette et al., Phys. Rev. A 61, 063418 (2000).

[22] G. Mitchison and S. Massar, Phys. Rev. A 63, 032105 (2001). 